\title{
3D Simulation Model of Lunar Crater Based on Single CCD Image
}

\author{
Junlin Wang ${ }^{1}$, Jiaqi $\mathrm{Li}^{1}$, Binbing Liu' ${ }^{1}$ Zhankai $\mathrm{Li}^{1}$, Xiaolin Tian ${ }^{1,2}$ \\ ${ }^{1}$ Faculty of Information Technology, Macau University of Science and Technology, Macau, China \\ ${ }^{2}$ Lunar and Planetary Science Laboratory/Space Science Institute, Macau University of Science and Technology, Macau, China \\ E-mail: zifuqiwu@gmail.com
}

\begin{abstract}
The paper gives an algorithm to simulate the crater topography from a single Charge Couple Device image. Refer to the Oren-Nayar reflectance model, several parameters of the lunar crater and constraint conditions have been added to this new algorithm, also the small craters have been enhanced in the frequency domain. Comparing with the Tsai's Shape from shading algorithm and the other new SFS algorithm, the simulation results of the new algorithm have shown more details of craters topography.
\end{abstract}

Keywords-Shape from shading, Lunnar crater geomorphic, Single CCD image, 3D Simulation

\section{INTRODUCTION}

Impact crater is an important and basic geomorphic feature on the lunar surface. Generally, great influences would be impacted on landing of the lunar landers by the surface relief of lunar landform. In order to insure the probe lands successfully, the digital elevation data of relief height around landing site is necessary for terrain matching guidance. Building the 3-D lunar map is of great importance for the mission planning and navigation of the probe[1-3].

Regularly, due to low sampling frequency of laser range finder on orbiter, the Digital Elevation Map (DEM) is often with low resolution for the lunar remote image. As we known, low resolution is no ideal for locating and guiding. But relatively, the resolution of the images obtained via CCD on orbiter is much higher than the one of the DEMs.

Via the Shape-from-shading (SFS in short) algorithm, elevation information can be obtained from the CCD images with high resolutions. As a common algorithm in the computer vision, SFS can be usually used in the 3D reconstruction. Proposed by B.K.P and Horn first[4], the brightness values of the image pixels are used as input of the chromaticity model function designed upfront in this algorithm, and solution of equation is elevation information. Due to the amount of unknown parameters is much more than the constraints, therefore more assumptions must be introduced in calculation process to reduce the range of the solutions set. There are many hypothesis and restrictions such as, differential properties; limitation of surface curvature, smooth degree can be introduced to obtain the solution with high accuracy. Based on this algorithm, image metric stereo is derived by $\mathrm{RJ}$ Woodham[5].

The input image has been analyzed in several aspects. Image input format is not required; all images can be done in this way. The effect depends on the situation. Fig. 1 has shown that the flows of us work. In order to restore a high- reducibility simulation result, the flows are necessary. The illumination needs to be considered before the geomorphology restoration. After obtaining the reflection model, the original geomorphology can be rebuilt by the gray-scale and gradient equation. In the other side, we need enhance the details in the frequency domain, and making the restoration results more approach to the original geomorphology.

The traditional methods cannot simulate the moon environment very well. A large number of samples have been analyzed. Through the analysis of the light, we have built a better simulation model. Large and small craters can be simulated at same time. the other knowing algorithms. It is unable to complete the task by other tradition algorithm.

\section{NEW ALGORITHM}

A series of operations have been used to simulate the crater topography from the single CCD image. The small craters have been enhanced in frequency domain. The new algorithm proposed gives a new way to restore the crater image which keeps more details of the craters. The flowchart of the new algorithm proposed has been shown in Fig. 1 as below.

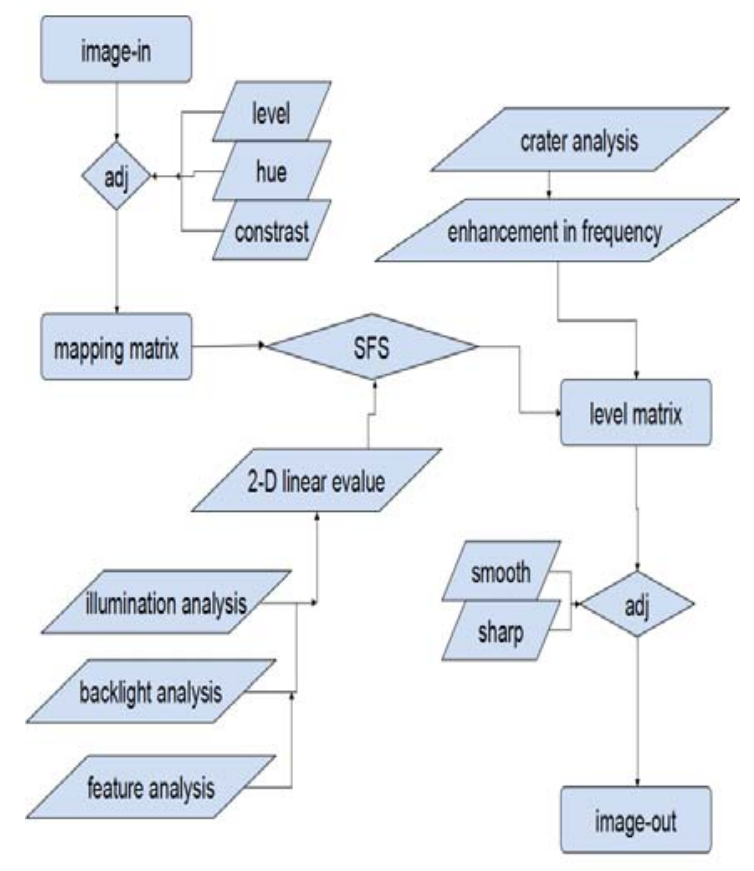

Fig. 1. The flowchart of the new algorithm proposed

\section{A. Illumination analysis}

The reflection equation was referred to the Oren-Nayar 
reflectance model [6] which is a famous model of the SFS. The formula in that model is as follow.

$$
\begin{gathered}
\operatorname{Lr}=\frac{\mathrm{p}}{\pi} * \operatorname{Cos}\left(\theta_{\mathrm{i}}\right)\left(\mathrm{A}+\left(\mathrm{B} * \max \left[0, \operatorname{Cos}\left(\Phi_{\mathrm{i}}-\Phi_{\mathrm{r}}\right)\right] * \operatorname{Sin}(\alpha) * \operatorname{Tan}(\beta)\right)\right) * \mathrm{E}_{0} . \\
\mathrm{A}=1-0.5 * \frac{\sigma 2}{\sigma 2+0.33} \mathrm{~B}=0.45^{*} \frac{\sigma 2}{\sigma 2+0.09} \\
\alpha=\max \left(\theta_{\mathrm{i}}, \theta_{\mathrm{r}}\right) \\
\beta=\min \left(\theta_{\mathrm{i}, 2}, \theta_{\mathrm{r}}\right)
\end{gathered}
$$

According to the environment on lunar, the illumination angle has been given as a variable value, it changes in the every input image. Also it has less parameter in the lunar environment than the earth environment.

It can be gotten from the system parameters, running state of satellite and environment parameters when we do the simulation. Otherwise, we have to given the Illumination lighting angle, through the crater morphology.

When the light's angel is less than the min- angle of incidence, it will make a dark area. We can't get the information in this area, but we can use our reflection model to set a constant angel value for these gray scales. With these values, the crater morphology can be simulated by this reflection equation, which is the result of the experiment.

Gray-scale corresponding angle $=\left\{\begin{array}{l}\text { Minus grade, Gray scale } \in(10,90) \\ \text { Up grade, Gray scale } \in(90,220) \\ \text { Bright area, Gray scale } \in(220,255)\end{array}\right.$

Also, the dark areas only take a little part in the whole image, so it doesn't effects a lot in the simulation results. Via building the model from the parameters, the relative between gray-scale corresponding angle can be found in the formula (According the formula 1).

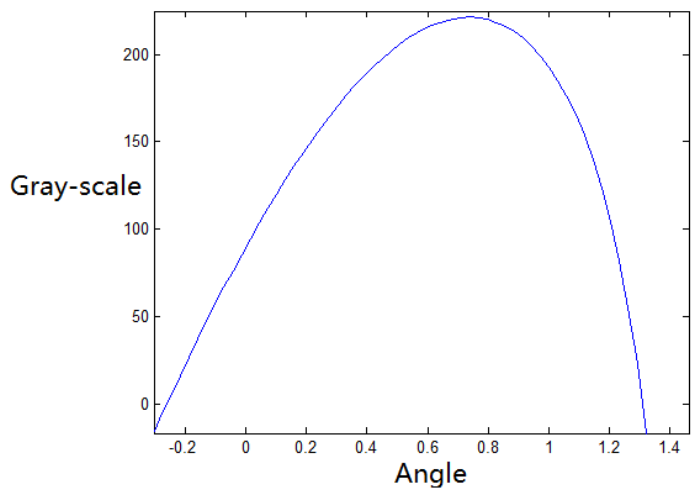

Fig. 2. The relationship between gray-scales and angles

According to the formula 1, a linear model can be built by these parameters .The Fig. 2 has been drawn by using the Matlab, it will gives the relation between the gray scales and the gradients for us. With the Fig. 2, the gradients of the gray scales can be found in it.

\section{B. Shape from shading}

The real elevation can be calculated by the Forward Elevation and Backward Elevation. The relationship between the Forward Elevation and Backward Elevation, Real
Elevation has been shown in the Fig. 3. And the formula 2 has been given.

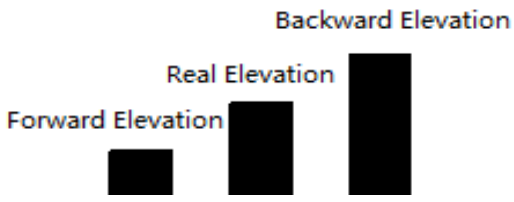

Fig. 3. The relationship of the elevations

According to the Fig. 3, we need to calculate these values during the elevation rebuilding, which are the forward elevation value, the real elevation value and the backward value. And the $\mathrm{k}$ is the sequence number of the pixel the algorithm processing.

Forward Elevation $\mathrm{k}=$ Backward Elevation $\mathrm{k}-1$ Backward Elevation ${ }^{k}=$ Forward Elevation $^{k}+\operatorname{Sin}\left(\right.$ angel $\left._{(k)}\right)$

Real Elevation $\mathrm{k}=$ Forward Elevation $^{\mathrm{k}}+\operatorname{Sin}\left(\right.$ angel $\left._{(\mathrm{k})}\right)$

Then, the crater morphology can be rebuilt from these formulas.

\section{Crater analysis}

Through the analysis of image, we obtained multiple sets of data. The main goal is to optimize shape of craters form obtained data. So, we focus on the crater land form restoration. In the analysis of a large number of experimental results, we found that, the algorithms for the reduction of large topography, the effect is clear. But for the small craters, which are usually in a 1-25 pixel size (just like the Fig. 4), the effect is not work well Sometimes those small craters even can't be shown in results.

In order to make the small craters more clearly in the simulation result, it needs to analyze the small craters in the frequency domain. Optimizing the reduction work on small craters to restore topography with more details, by strengthen the small crater in frequency domain.

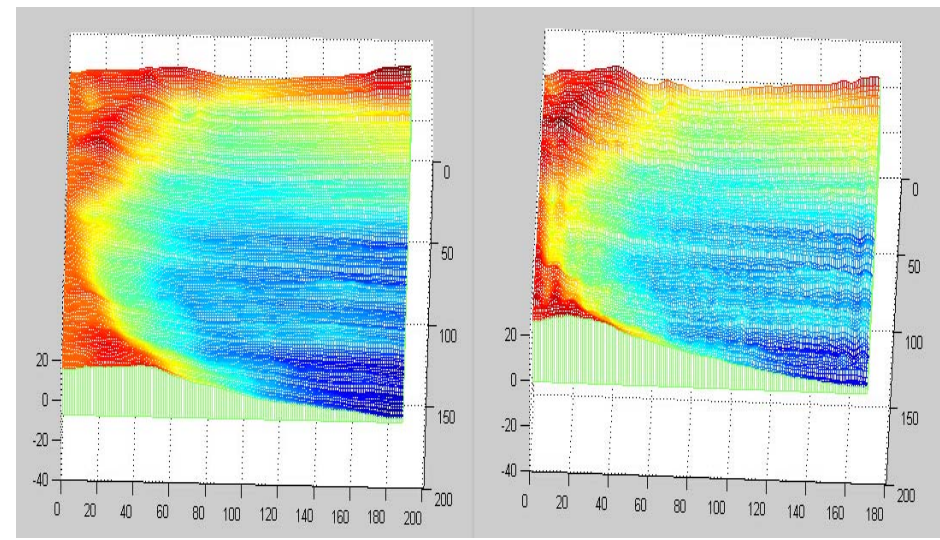

(1). Before enhancement (2). After enhancement

Fig. 4. Effect of enhancing in the frequency domain 
The (1) of the Fig. 4 is the simulation result which hasn't been processed in the frequency domain. And the (2) of the Fig. 4 is the simulation result which has already been processed by enhancement in frequency domain. Comparing with the (1) of the Fig. 4, (2) of the Fig. 4 keeps the more details of the small craters. The geomorphology of the small craters can be showed clearly in the (2) of the Fig. 4.

\section{EXPERIMENTAL RESULTS AND DISCUSSION}

In this paper, the single CCD images of lunar craters have been random selected as the training samples and the testing samples. Two training samples and ten testing samples have been selected to test this new algorithm. Comparing with the original image, the quality of simulation algorithm can be proposed can be viewed clearly. The results of new algorithm have been compared with the classic Shape-from-shading algorithm of Tsai[7] and the other new algorithm[8].

In this experiment, the direction of the light source has been given as $30^{\circ}$. So we stipulate the darkness part of the craters must on the left of the whole image, by spinning the single CCD images, the image can be adjusted in place. The transverse tectonic begins from the left of the single CCD images.

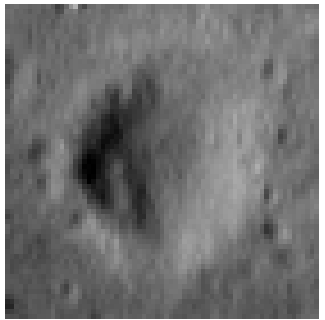

Fig. 6. Original Testing Image 1

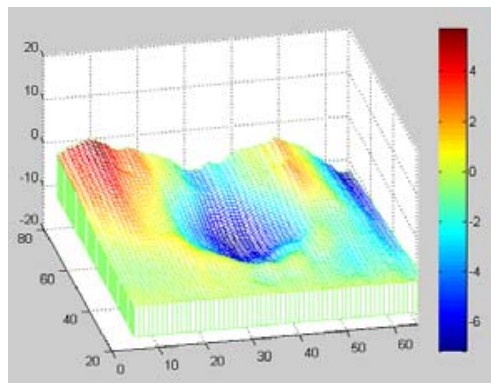

Fig. 7. Processed by the algorithm proposed

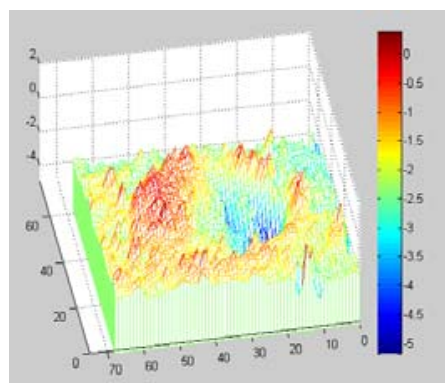

Fig. 8. Processed by the algorithm of Tsai algorithm

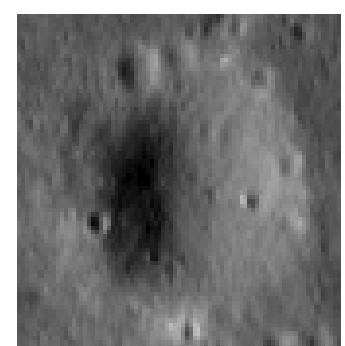

Fig.9.Original Testing Image 2

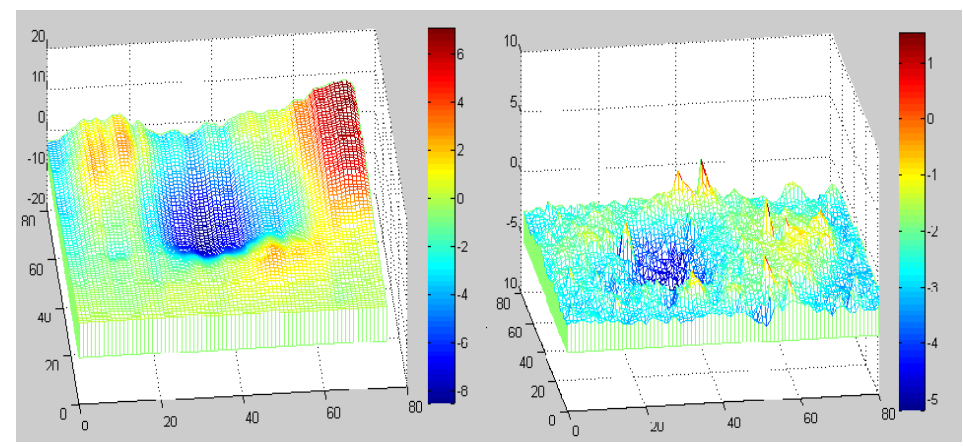

Fig. 10. Processed by the Fig. 11. Processed by the algorithm in this paper Tsai algorithm

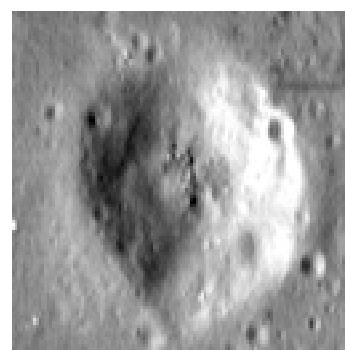

Fig.12.Original Testing Image 3

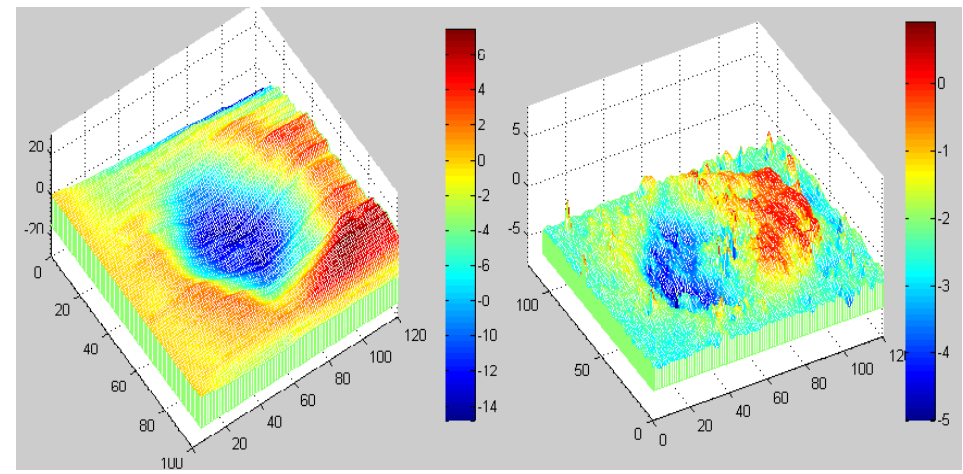

Fig. 13. Processed by the algorithm in this paper

Fig. 14. Processed by the Tsai algorithm

According to the testing results, the simulation model of the algorithm in this paper can almost rebuild the geomorphology of the crater. And the Tsai algorithm did not do well in the dark part, it can only simulated half of the whole crater. The result has been shown in the Fig. 8. The Tsai algorithm can't rebuild the part which is dark success, because it hasn't considered the source's angel and the dark part's gradient. 
This problem led to a result which is distortion. In the other side, the result of the algorithm in this paper can rebuild the geomorphology clearly and the Tsai algorithm has lost some information of the crater geomorphology.

Also the algorithm in this paper has been used to compare with the new SFS algorithm in the other paper [7]. We reference the sample and the result in that paper and contrast the result with our algorithm.

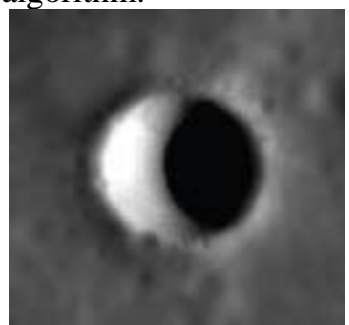

Fig. 15. Original Testing Image 4

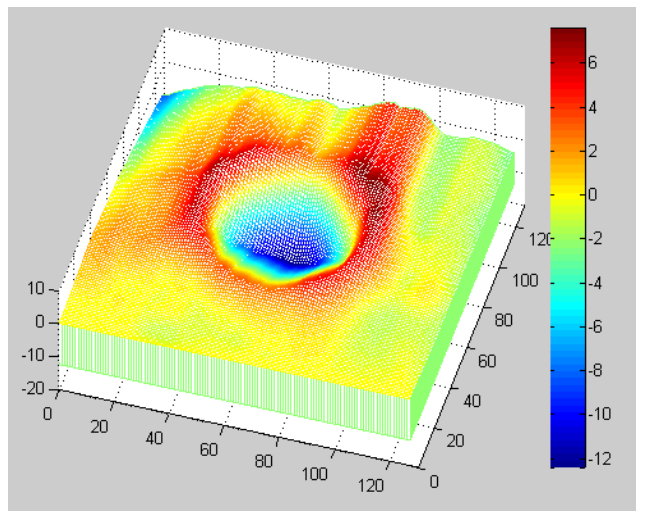

Fig. 16.Algorithm in this paper

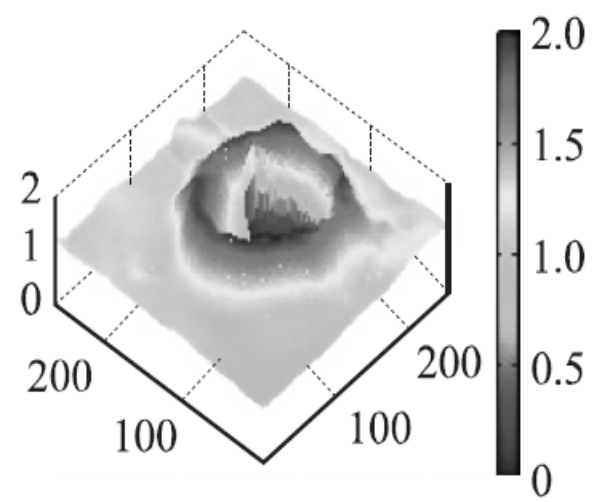

Fig. 17. Algorithmby Mr.xu [8] 2014
The result processed by the algorithm in this paper gives a better geomorphology restoration, which has been shown in the Fig. 16 and the Fig. 17. Our algorithm rebuilt the bonder of the crater more similar to the original CCD image.

\section{CONCLUSION}

This paper has referred to the Oren-Nayar reflectance according to the lunar environment, which makes the model more suitable for the lunar surface. The new algorithm also enhances the small craters in the frequency domain. The simulation results are better than the existing algorithm before, the new algorithm can show more details of the craters, and can work on the single CCD image. To a certain extent, it resolves some problems of the surface relief landform.

In the other side, the new algorithm only can rebuild the darkness of the crater according to an estimated value. And the other limitation of this algorithm is that the illumination angels should be determined before the simulation. We will try to remove this limitation in our future works.

\section{ACKNOWLEDGMENT}

This work is supported by the Science and Technology Development Fund of Macao (No. 059/2013/A2).

\section{REFERENCE}

[1] Jia Y, JX Chen, HG Zhang. Key Technology Analysis of Lunnar Rover, Spacecraft engineering, 2006,16(03):54-58.

[2] Williams DR, Grayzock EJ. The lunar data projectrestoration of Apollo data for future lunar exploration.Lunar and Planetary Science, 2006, 37(06):1187-1224.

[3] K Xu, Y Zhou, JS Li, F Teng, LV Liang. A Lunnar Landing Area Select Method Based on Single Image. Journal of Geomatics Science and Technology, 2012, 29(2): 127-130.

[4] BKP Horn, The variational approach to shape from shading MJ Brooks. Computer Vision, Graphics, and Image Processing, 1986

[5] RJ Woodham. Gradient and curvature from the imagemetric-stereo method including local confidence estimation. JOSA A, 1994, 11(11): 3050-3068.

[6] LB Wolff, SK Nayar, Oren M. Improved diffuse reflection models for computer vision. International Journal of Computer Vision, 1998, 30(1): 55-71.

[7] PS Tsai, M Shah. A fast linear shape from shading. Computer Vision and Pattern Recognition, 1992. Proceedings CVPR'92, 1992 IEEE Computer Society Conference on. IEEE, 1992: 734-736.

[8] XC Xu, AG Xu, SC Liu, ZQ Xu. 3D reconstruction algorithm for lunar crater based on new SFS. Science of Surveying and Mapping. 2014, 9:01 